\title{
Biogeografía de.las planarias de aguas dulces (Platelminthes; Turbellaria; Tricladida; Paludicola) en España. Datos preliminares.
}

\author{
Jaume Baguñá; Emili Saló i Rafael Romero
}

Departament de Genètica, Facultat de Biologia, Universitat de Barcelona, Diagonal 645, Barcelona-28

\author{
SUMMARY \\ BIOGEOGRAPHY OF FRESHWATER PLANARIANS (PLATYHELMINTHES; \\ TURBELLARIA; TRICLADIDA; PALUDICOLA) OF SPAIN. PRELIMINARY DATA.
}

The geographical distribution of freswater planarians of Spain has been studied from old and new literature data and from recently collected material. Ten epigean species and three hipogean (subterranean) species have been found throughout the 128 localities studied. All the epigean species belong to the Dugesiid and Planariid families; no Dendrocoelidae having been found so far.

Although the species and the geographical pattern found are similar to the published european records, some peculiar characteristics like the overwhelming presence of runningwater species over still-water species; and the hipogean temporal habits of some epigean species, are discussed. The modalities of dispersal throught continental waters and island colonization are also discussed.

Finally a sketch for a future biogeographical programme is given, where the ned to study the karyotypes and the pattern of soluble and structural proteins for each population is again stressed in order to diagnose correctly some of the present and new species and to measure their phyletic distance.

\section{INTRODUCCION}

Desde la inicial, y ya clásica, monografía de los tríclados del Norte de Francia (HALLEZ, 1894), varias han sido las revisiones publicadas sobre la sistemática y distribución de estos organismos en Europa (países nórdicos: LUTHER, 1961; Países Bajos: HARTOG, 1962; Luxemburgo: HOFFMANN, .1964b; Francia: PATTÉE \& GOURBAULT,
1981; Reino Unido: REYNOLDSON, 1967; BALL \& REYNOLDSON, 1981; 1talia: BENAZZI, 1955, Yugoeslavia: STANKOVIC, 1960, Rusia: ZABUSOVA, 1970), América del Norte (KENK, 1976) y Japón (KAWAKATSU, 1965). Existen además diversos trabajos que tratan sobre la distribución y sistemática de los tríclados en regiones más circunscritas, y en contrapunto, se han publicado índices de distribución en grandes re- 
giones y áreas continentales (Europa: Limnofauna Europaea, DAHM \& GOURBAULT, 1978; Africa: YOUNG, 1976; Asia: BALL, 1970; América del Norte: KENK, 1976; América del Sur y Central: BALL, 1971; Australia: BALL, 1974c) e incluso un indice mundial de géneros y especies (KENK, 1974).

Como ha sucedido, y sucede aún, en tantos campos de la faunística, el conocimiento de la sistemática y distribución de estos organismos en España es muy imperfecto. No hay monografía o trabajo alguno que trate específicamente sobre la distribución geográfica y la sistemática de este grupo en España, y lo que es peor, los pocos trabajos de faunistica o limnología general que mencionan géneros o especies del grupo son generalmente antiguos e incompletos, y muy a menudo de diagnóstico erróneo. El reciente interés en los temas de Ecología y Limnología, y consecuentemente de los estudios de catalogación de especies de diversos habitats, no ha supuesto desgraciadamente estímulo alguno para un mejor conocimiento del grupo. De aquí, que los autores de este trabajo tuvieran el dudoso honor de publicar el primer catálogo sistemático y geográfico de planarias de aguas dulces en un área relativamente restringid a de la peninsula ibérica: Catalunya e islas Baleares (BAGUÑÀ et. al., 1980).

Para paliar parcialmente este vacío, el trabajo que sigue resume, a partir de una revisión crítica de la bibliografía publicada hasta la fecha y de recolecciones recientes realizadas por diversos autores, el estado actual de los conocimientos sobre la biogeografía de los tríclados de agua dulce en España.

\section{METODOS}

Los métodos de recolección, observación y conservación de ejemplares han sido descritos ya anteriormente (BAGUÑ̀̀ et. al., 1980) Las revisiones de BALL \& REYNOLDSON (1981) y de PATTÉE \& GOURBAULT (1981) son especialmente útiles al respecto.

\section{CLASIFICACION ACTUAL}

Las planarias de aguas dulces constituyen el infraorden PALUDICOLA del filum PLATYHELMINTHES, clase TURBELLARIA orden SERIATA, suborden TRICLADIDA. Según la nueva clasificación propuesta por BALL (1974), y aceptada hoy en día por la mayoría de autores, los PALUDICOLA constan de 3 familias: DUGESIIDAE, PLANARIIDAE y DENDROCOELIDAE, esta última subdividida en 2 subfamilias: KENKIINAE y DENDROCOELINAE.

Los criterios de identificación y diagnóstico, así como una clave sistemática para clasificar correctamente las especies de este grupo de organismos ha sido ya publicada (BAGUÑ̀̀ et. al., 1980). Otras clasificaciones útiles son las de REYNOLDSON (1967), BALL \& REYNOLDSON (1981) y PATTEEE \& GOURBAULT (1981). En la tabla I se incluyen las características para diferenciar las familias y subfamilias.

\section{RESULTADOS}

\section{ESPECIES PRESENTES EN ESPAÑA}

Basándonos en las citas bibliográficas, en el trabajo ya publicado sobre distribución de planarias de aguas dulces en Catalunya y $\mathrm{Ba}$ leares (BAGUÑ̃̀ et. al., 1980), y en nuevas localidades encontradas con posterioridad a este trabajo, podemos confeccionar un catálogo de especies de tríclados de aguas dulces presentes en España (tabla II).

De las 13 especies presentes, 10 son epigeas y 3 hipogeas. Estas últimas pertenecen a un dominio muy insuficientemente estudiado en España por lo que a tríclados se refiere. De aquí que no tratemos de ellas en este trabajo, aunque damos a continuación las citas que se conocen hasta el presente: (Phagocata (Atrioplana) racovitzae, en una napa aluvial del rio Henares, Madrid (GOURBAULT, 1971a); Crenobia alpina anophthalma, de gruta del manantial de Gorbea en Cigoitia, provincia de Alava (DE BEAU- 
TABLA I. Características diferenciales de las familias y subfamilias del infranden PALUDICOLA.

Familia DUGESIIDAE, Ball Generalmente pigmentados. Cabeza triangular o ligeramente redondeada, sin órganos adhesivos: Siempre presentan ojos. Musculatura interna de la faringe en dos capas definidas. Oviductos que desembocan separadamente (o formando un conducto común) en el canal de la bolsa copuladora. Huevos (cocoons) esféricos y pedunculados. Distribución: cosmopolita.

Género tipo: Dugesia (Girard)

Géneros más característicos: Dugesia, Cura.

Especies europeas más comunes: Dugesia (S.) Zugubris, Dugesia (S.) poLychroa, Dugesia (D.) gonocephala, Dugesia (G.) tigrina

Familia PLANARIIDAE, Stimpson, emend.

Pigmentados o blancos. Cabeza truncada o redondeada, nunca triangular. ojos generalmente presentes. Musculatura interna de la faringe en dos capas definidas. Oviductos formando un conducto común que desemboca, independientemente del canal de la bolsa copuladora, en el atrio genital. Adenodáctilos generalmente presentes. Huevos (cocoons) esféricos u ovalados sin pedúnculo. Distribución: holártica y parte de la región oriental.

Género tipo: Planaria (Müller)

Géneros más característicos: Planaria, Polycelis, Phagocata, Crenobia. Especies europeas más comunes: Polycelis nigra, Polycelis felina, Crenobia alpina, Phagocata vitta, Planaria torva.

Familia DENDROCOELIDAE, , Hallez

Presentan generalmente un órgano anterior músculo-glandular. Oviductos formando un oviducto común que desemboca en el atrio genital, independientemente del canal de la bolsa copuladora. Huevos esféricos sin pedúnculo. Distribución: holártica y región oriental.

Género tipo: Dendrocoelum (Oersted)

Géneros más característicos: Dendrocoelum, Kenkia

- Subfamilia KENKIINAE, BaIl

Generalmente hipogeos, no pigmentados y ciegos. Musculatura interna de la faringe en dos capas definidas. Presentan adenodáctilos. Testículos situados prefaríngeamente. Distribución: América del Norte y Asia Oriental.

Género tipo: Kenkia

Géneros más característicos: Kenkia, Sphalloplana

- Subfamilia DENDROCOELINAE, Ball

No pigmentados y generalmente con ojos. Musculatura interna de la faringe de tipo mezclado. Presentan adenodáctilos. Testículos situados a lo largo de todo el cuerpo. Distribución holártica.

Género tipo: Dendrocoleum

Géneros más característicos: Dendrocoelum, Bdellocephala, Rectocephala. Especies europeas más comunes: Dendrocoelum lacteum, Bdellocephala punctata. 


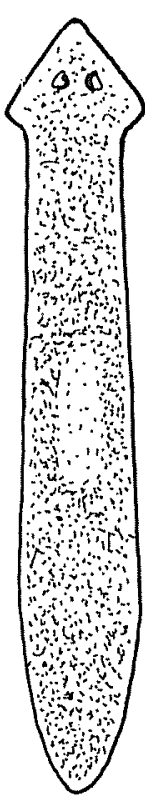

a

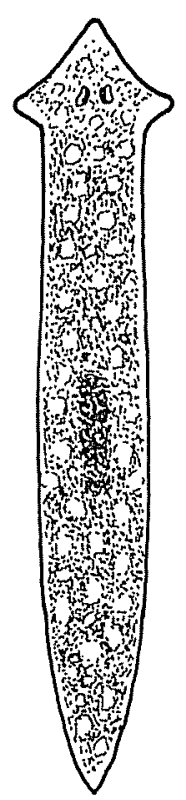

b

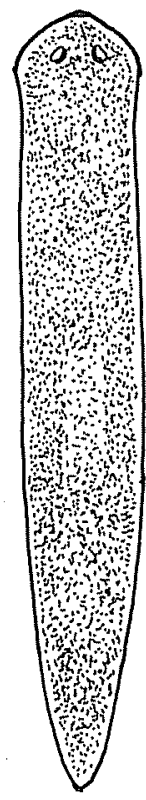

C

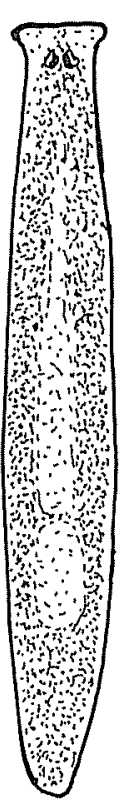

d

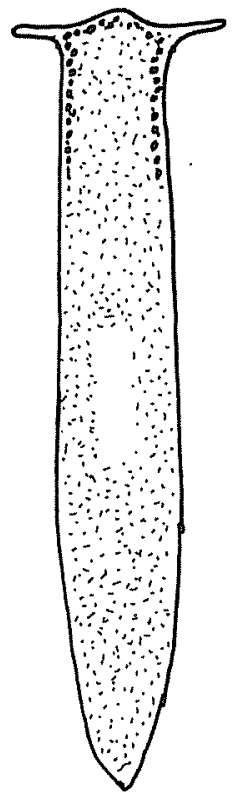

e
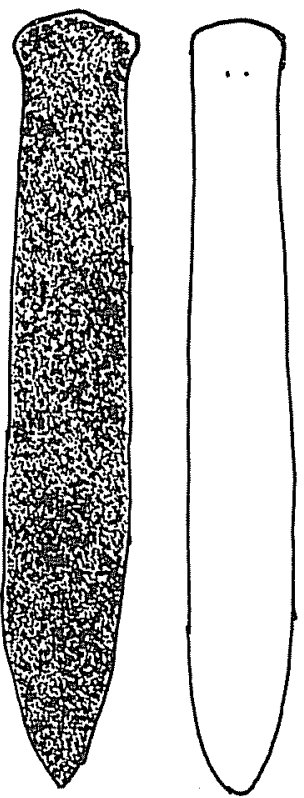

f

Fig. 1. Representación gráfica (vista dorsal) de las especies de planarias de aguas dulces presentes en España. Las especies están representadas a diferente escala. Para conocer la longitud típica, ver BAGUÑ et al. (1981).

\section{Fann. Dugesiidae}

a Dugesia (D) gonocephala Dugesia (D) iberica Dusesia (D) sicula b Dugesia (G) tigrina

c Dugesia (S) polvchroa Dugesia (S) mediteranea
Fam. Planaritalae
d Crenohia alpina
f Polycelis nigra
e Polveclis felina
a Phagocata vitta

aún por estudiar. De aquí, que la distribución presentada haya de tomarse, hoy por hoy, como algo enteramente provisional. De toda manera, y a grandes rasgos, la distribución de las planarias epigeas en España muestra un patrón similar al patrón general europeo, con las especies estenotermas de aguảs frias (Crenobia alpina, Polycelis felina) situadas en los lagos alpinos y tramos superiores de los ríos, las especies euritermas y menos reófilas en zonas intermedias (Dugesia $(D)$ gonocephala, Dugesia ( $G$ ) tigrina), y finalmente las especies de aguas quietas o de tramos inferiores de los ríos y lagos costeros (Dugesia (S) polychroa, Dugesia (S) mediterránea y Polycelis nigra). Sin embargo, existen ciertas peculiaridades en la distribución que merecen un comentario aparte.

De las 128 localidades descritas, 108 corresponden a tan solo 3 especies: Dugesia $(D)$ gonocephala, Dugesia (G) tigrina y Polycelis felina, las tres, propias de hábitats más o menos reófilos. Por contra, las especies de aguas quietas (lagos, canales y tramos inferiores de los ríos) como Dugesia (S) poly. chroa, Dugesia (S) lugubris, Dendrocoelum. 


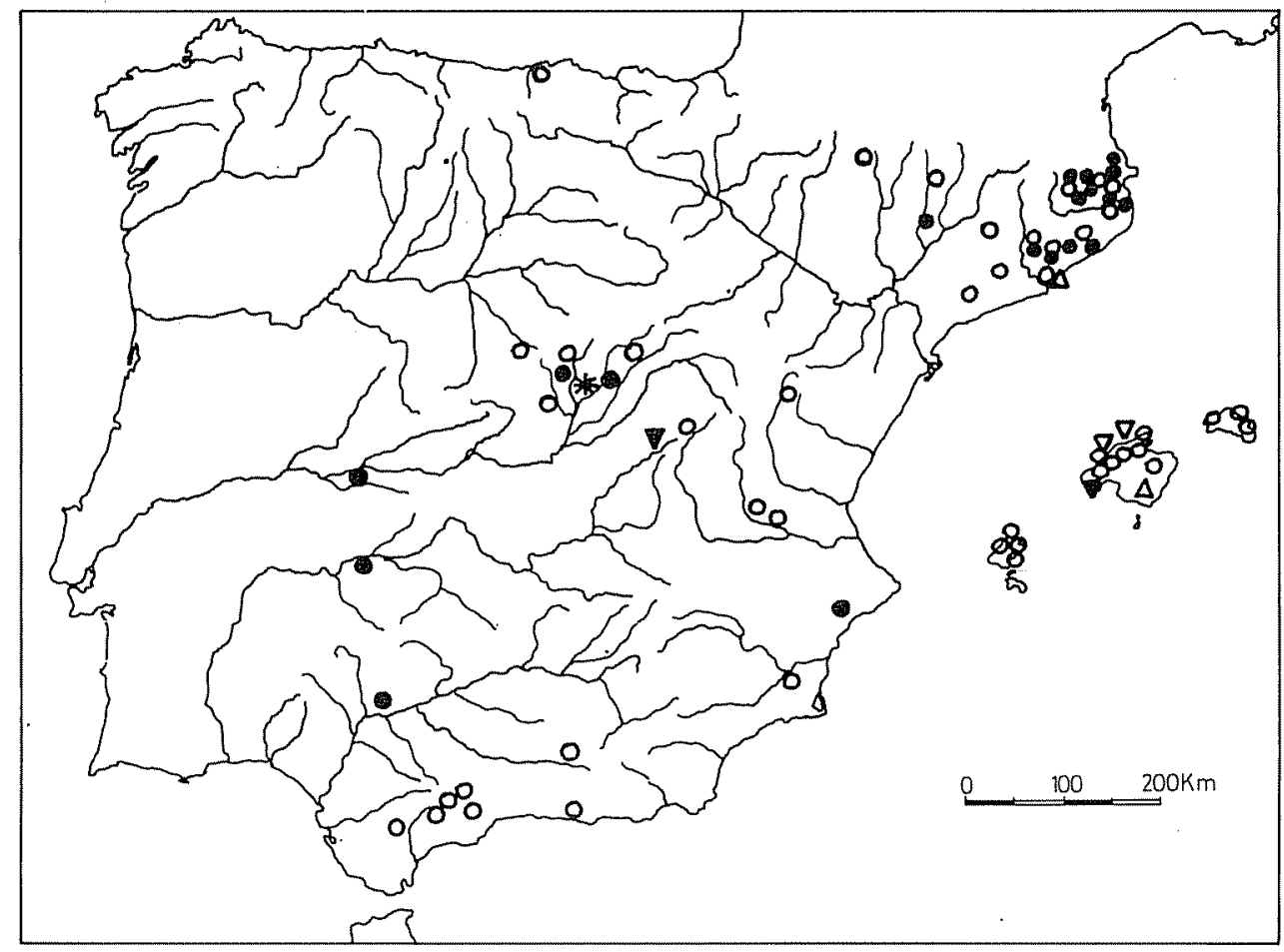

Fig. 2. Mapa de distribución de las distintas especies de planarias de aguas dulces de la familia Dugesildae en España. (Se incluyen también las localidades mencionadas en la bibliografía).

$$
\begin{aligned}
& \text { Dugesia (D) gonocephala } \\
& \text { Dugesia (D) iberica } \\
& \text { Dugesia (D) sicula }
\end{aligned}
$$

lacteum, Polycelis nigra, Polycelis tenuis, y Planaria torva son muy poco abundantes o no han sido aún detectadas (caso de $P l$. torva, Polycelis tenuis, y probablemente de D. lacteum y Dugesia (S) lugubris).

A nuestro entender, la explicación más verosimil de esta característica radica en la relativa escasez de lagos naturales (no alpinos), canales, y grandes ríos en la Peninsula, hábitats donde predominan estas últimas especies, y en los que son dominantes en la mayoria de países europeos estudiados. De aquí, que sería muy interesante un estudio sistemático de los tríclados de aguas dulces en los escasos lagos naturales no alpinos de la península, así como un estudio de los numerosos pantanos existentes para establecer si
- Dugesia (G) tigrina

* Dugesia (S) polvchroa

$\triangle$ Dugesia (S) mediteranea

han supuesto un nuevo hábitat para la expansión de estas especies.

\section{DOMINIO HIPOGEO DE Crenobia alpina}

Crenobia alpina es la especie europea propia de hábitats fríos más o menos reófilos (lagos alpinos, cabeceras de los ríos, y lagos de planicie en regiones septentrionales). En la mayoría de países europeos, especialmente los septentrionales, es relativamente abundante, compitiendo con Polycelis felina en determinados tramos de los ríos. En la península ibérica, su distribución es exigua 


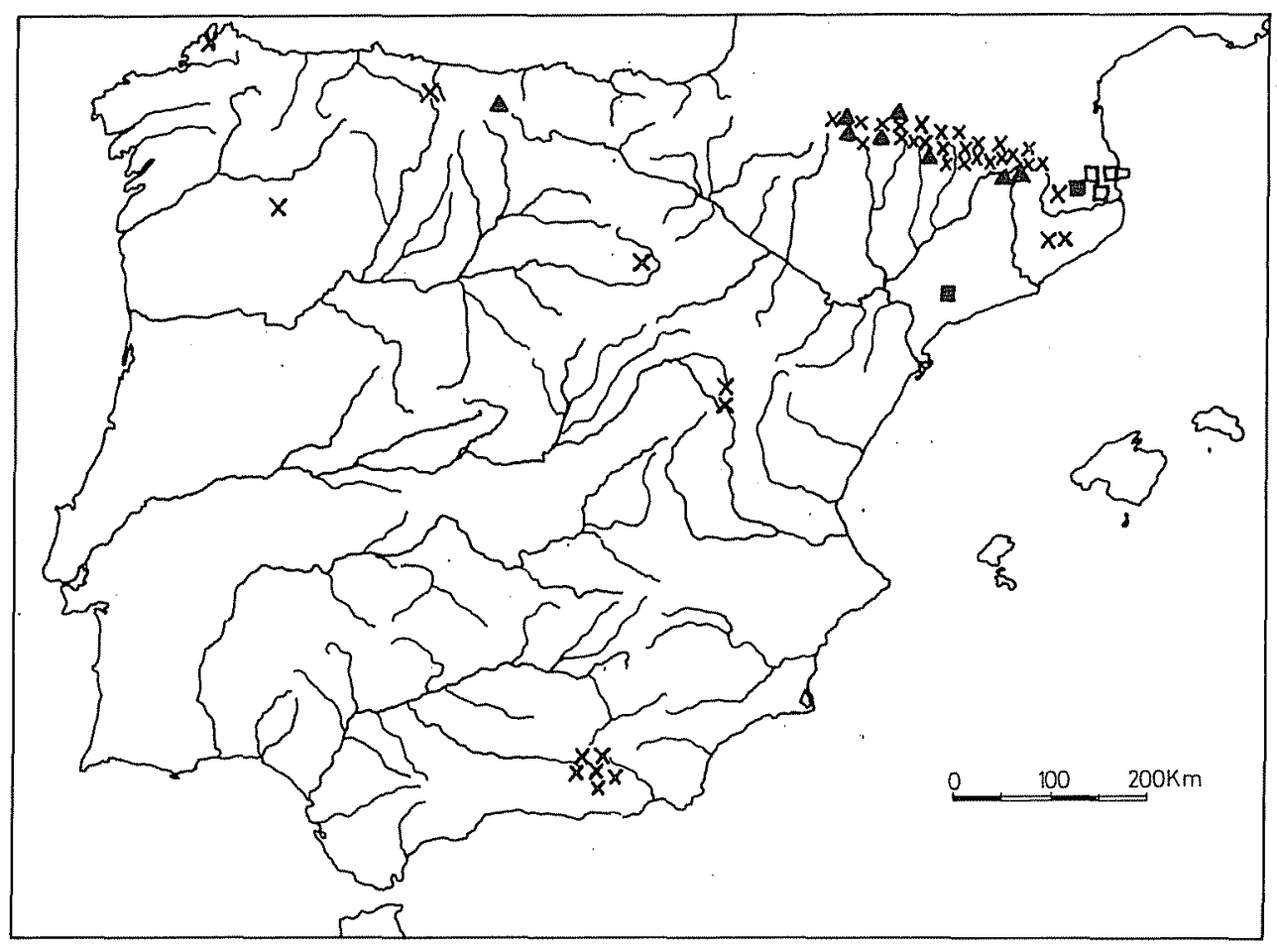

Fig. 3. Mapa de distribución de las distintas especies de planarias de aguas dulces de la familia Planariidae en España. (Se incluyen también las localidades mencionadas en las referencias bibliográficas).
A Crenobia alpina
ㅁ Polycelis nigra
$\mathrm{X}$ Polyeclis felina
Phagocata pitta

(8 localidades frente a las 43 de Polycelis felina) siendo más frecuente en aguas subterráneas, napas fluviales y fuentes que en hábitats claramente epigeos (lagos y rios). En estos últimos, su presencia queda circunscrita a lagos y tramos fluviales por encima de los 2.000-2.200 mts, y aún en estas zonas compite a menudo con Polycelis felina.

Una posible explicación a este predominio hipogeo de Crenobia alpina en España radicaria en que esta especie, estenoterma y de aguas frías, tiene muy pocos hábitats epigeos en los que puede mantenerse sin competencia (principalmente de Polycelis felina). De aqui, que su distribución actual y la invasión del hábitat hipogeo sean el resultado de la regresión en la distribución de las especies alpinas desde la última glaciación. Es interesante señalar en este con- texto que en Sierra Nevada y en alturas desde 2.200 a $2.800 \mathrm{mts}$, no se detecta Crenobia alpina siendo Polycelis felina la especie dominante.

\section{LA DISTRIBUCIÓN DISJUNTA DE Dugesia $(S)$ mediterránea $y$ Dugesia (D) sicula}

La distribución de Dugesia (S) meditemánea, circunscrita a una localidad de la península (Barcelona) y una localidad de las islas Baleares (Mallorca), junto a las conocidas ya anteriormente en las islas de Córcega, Cerdeña y Sicilia, plantean el problema de dar una explicación coherente a esta distribución $\tan$ disjunta. Un problema similar 


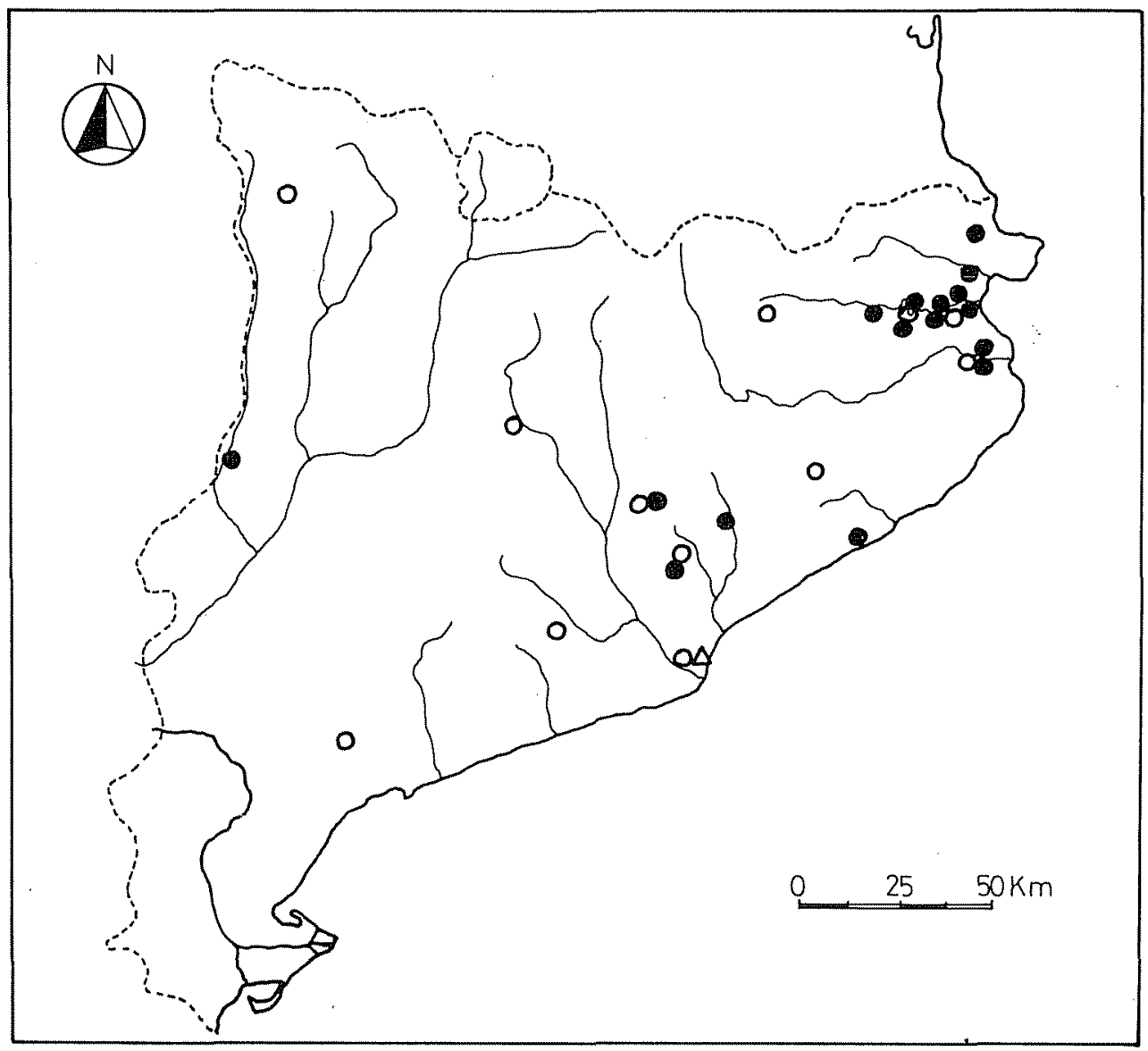

Fig. 4. Mapa de distribución de las distintas especies de planarias de aguas dulces de la familia Dugesiidae en Catalunya. (Se incluyen también las localidades mencionadas en la bibliografía).

$\Delta$ Dugresia (D) gonocephala

- Dugesia (G) tigrina

$\triangle$ Dugesia (S) mediterranea

se plantea con Dugesia (D) sicula, del grupo "gonocephala", hoy en día descrita en Sicilia, isla de Elba y Mallorca.

Aunque es posible imaginar diversas interpretaciones, la hipótesis más razonable sugiere que esta distribución notablemente disjunta sería el resultado de la fragmentación de una masa continental tirrénica (en la que originalmente se situaban estas poblaciones) y la subsiguiente rotación y migración de las microplacas resultantes en el:Mediterráneo Occidental desde el Oligoceno al Plioceno (BAGUÑ̇̀ el. al., 1981). Esta hipótesis, aplicada por estos autores a Dugesia $(S)$ mediterránea, podemos aplicarla asimismo a
Dugesia (D) sicula, aunque serían necesarios estudios de cariología comparada (a poder ser empleando técnicas de bandeo de cromosomas premetafásicos) y estudios comparativos del patrón de proteínas estructurales y enzimas entre las diversas poblaciones para discernir claramente entre las diversas hipótesis propuestas.

\section{AUSENCIA DE LAS ESPECIES DE LA FAMILIA PLANARIIDAE EN LAS ISLAS BALEARES}

Aunque la distribución de los tríclados de agua dulce en las islas Baleares no ha estado 


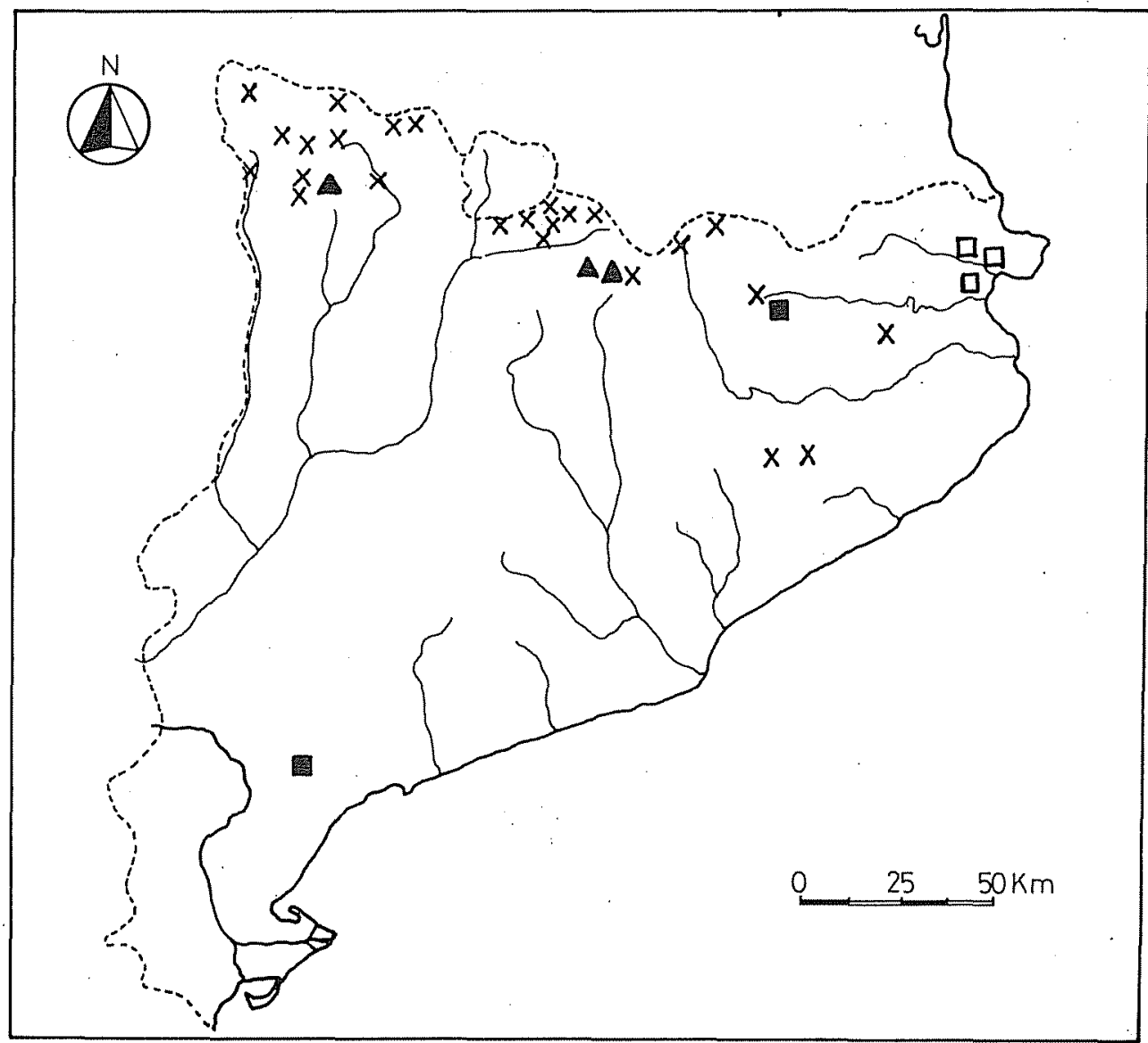

Fig. 5. Mapa de distribución de las distintas especies de planarias de aguas dulces de la familia Planariidae en Catalunya. (Se incluyen también las localidades mencionadas en la bibliografía).
A Crenohia alpina
口 Polveclis nigra
$\mathrm{X}$ Polvcelis felina
- Phagocata vitta

sujeta a un estudio en profundidad, en las más de 20 localidades estudiadas hoy en día no se han detectado especies de la familia Planariidae. Por contra, los representantes de la familia Dugesiidae son harto frecuentes. En principio, las islas Baleares (especialmente Mallorca) presentan hábitats que podrían acomodar a Polycelis felina, Polycelis nigra y Planaria toma.

Las razones de esta ausencia (que recordemos de nuevo como enteramente provisional hasta disponer de un estudio exhaustivo) pueden ser varias y han sido discutidas ex- tensamente por BAGUÑA et. al., (1981). Entre las hipótesis expuestas, estos autores sugieren como más verosímil que dado que la familia Planariidae es filogenéticamente más reciente que la familia Dugesiidae, el proceso de fragmentación y migración de microplacas habría sucedido en un período anterior a la constitución de esta familia. De todas maneras, explicaciones de otra indole, como son el carácter estacionalmente cálido de las islas que no favorecerían el ciclo vital de estas especies (especialmente de Polycelis felina), pueden haber jugado un papel deter- 
minante. Sin embargo, la isla de Mallorca, al igual que alguna de las Tirrénicas (especialmente la de Córcega) presentan cadenas montañosas con rios y riachuelos en que estas especies podrían estar përfectamente adaptadas.

\section{Sobre la PResencia de Dugesia (D) gonocepbala EN LAS IsLAS CANARIAS}

En un reciente artículo, BENAZZI \& DERI (1980) describen una población de Dugesia (D) gonocephala en San Nicolás (Gran Canaria). La presencia de esta especie en las islas Canarias plantea, al igual que en el caso de las islas Baleares y. las Islas tirrénicas, la modalidad de su poblamiento. Es sabido que las planarias de aguas dulces son organismos frágiles, carentes de huevos o estadios embrionarios resistentes, lo que dificulta grandemente su dispersión a través de los agentes dispersivos clásicos (aves, viento, corrientes, etc, $\ldots$ ). De aquí que diversos autores consideran a estos organismos como buenos indicadores paleogeográficos (BALL, 1974), y que los estudios más recientes de biogeografia de este grupo expliquen los patrones de distribucion en términos de deriva continental $y$ fragmentación y migración de microplacas (BALL, 1977b; BAGUÑ̃̀ et. al., 1981). Sin embargo, se conocen casos fehacientes de introducción de especies debido a la actividad humana. Este fenómeno, que algunos autores (REYNOLDSON, com. pers) consideran cada vez más frecuente, podría explicar así mismo el poblamiento insular de determinadas especies de aguas dulces.

En el caso concreto de Dugesia (D) gonocephala en Gran Canaria, hemos de señalar que un punto crucial: el origen geológico de las islas es aún foco de intenso debate. Para algunos autores, su origen es claramente volcánico. Si así fuera, el poblamiento a través del hombre u otro agente externo (?) sería la única alternativa viable. Por contra, otros autores sugieren que su. origen es el resultado (al menos parcial) de la fragmentación de la placa norteafricana. Si así fuera, la hipótesis de una dispersión por fragmentación y migración de microplacas, similarmente a lo propuesto para el poblamiento tirrénico, sería harto coherente, aunque no pudiera descartarse una reciente introducción humana.

Dado que ambas interpretaciones son factibles, tan solo un estudio detallado del cariotipo y del patrón proteíco de poblaciones insulares y continentales (Dugesia $(D)$ gonocephala está presente en el Norte de Africa) podría discriminar entre ambas hipótesis.

\section{PERSPECTIVAS DE LOS ESTUDIOS BIOGEOGRAFICOS}

\section{EL PROGRAMA IDEAL}

El adecuado conocimiento de la biogeografía de planarias de aguas dulces en España requiere en primer lugar un esfuerzo intensivo en completar el mapa de distribución de especies, especialmente en aquellas zonas (muy amplias todavia) de las que carecemos aún de datos.

Junto a este esfuerzo prospector, es necesario definir con precisión y distinguir adecuadamente las especies verdaderas de las meras variantes morfológicas o cariológicas (biotipos). Ello requiere estudios extensivos y continuados de morfología (especialmente del aparato reproductor), cariología, y muy especialmente de los patrones de proteinas estructurales y enzimáticas. Este tipo de estudios son. a menudo insuficientemente atendidos por taxónomos y ecólogos.

Alcanzado este estadio de conocimientos es cuando podemos tratar de establecer una filogenia coherente del grupo. Esta filogenia, junto a los datos tectónicos y climáticos del área de interés han de conducir a formular una hipótesis coherente sobre la historia biogeográfica de este grupo de organismos en la península ibérica.

No es preciso señalar que estamos aún muy lejos de poder emprender un programa de este tipo. En el ínterin, y sin olvidar este ambicioso objetivo, es preciso atender problemas a la vez más asequibles y reales, de los que entresacamos; los de diagnosis 
taxonómica, las rutas de dispersión y los de competencia.

\section{PROBLEMAS DE DIAGNOSTICO TAXONOMICO}

Existen en planarias de aguas dulces tres ejemplos paradigmáticos que instruyen muy adecuadamente sobre la necesidad de abordar en toda su extensión estudios.cariológicos y de anatomía comparada del aparato reproductor para llegar a un buen diagnóstico. Un primer ejemplo lo constituyen las especies del denominado "grupo lugubris" estudiadas por BENAZZI y su escuela (BENAZZI \& BENAZZI-LENTATI, 1976). Estas especies, de aspecto externo muy similar, comprenden hoy en día 9 biotipos distintos que se agrupan en 5 posibles especies (3 de ellas ya definidas: Dugesia (S) polychroa, Dugesia (S) lugubris, y Dugesia (S) mediterránea) distinguibles únicamente a nivel cariológico. Otro ejemplo lo constituyen las especies del denominado "grupo gonocephala" estudiado también por BENAZZI y otros autores (BENAZZI \& BENAZZI-LENTATI, 1976; DE VRIES \& BALL, 1980). Estas especies tienen asimismo un aspecto externo muy similar, y sólo un estudio a fondo de su aparato reproductor $y$ de su cariotipo ha permitido detectar más de 12 biotipos que podemos agrupar hoy en día en 8 especies definidas. Un último ejemplo lo constituye la clásica distinción entre Polycelis tenuis y Polycelis nigra, de morfología externa muy similar, pero fácilmente distinguible por su cariotipo y la morfología del aparato reproductor.

Bajo esta perspectiva parece pues evidente que un estudio adecuado de la distribución de estos organismos ha de contemplar necesariamente un adecuado estudio de su cariotipo y un estudio comparado del aparato reproductor en cada una de las poblaciones sujeto de estudio.

\section{LAS RUTAS DE DISPERSION}

Otro aspecto biogeográfico de interés, y prácticamente negligido en planarias, son las posibles rutas de dispersión de estos organismos en aguas continentales. Un ejemplo reciente de introducción y expansión de unạ especie americana en Europa puede servir de ejemplo. En 1925 se detectan en Alemania ejemplares de la especie americana Dugesia $(G)$ tigrina, muy probablemente introducida a través del comercio de plantas acuáticas y acuarios. En 1946 se detecta su presencia en los alrededores de París, en 1966 en Provenza y Aquitania, y a principios de 1970 en ríos del norte de Girona (España). Con posterioridad, se ha detectado en numerosas localidades de la península.

Aunque puede especularse que la actividad humana ha jugado un papel importante en la facilidad de dispersión, el hecho de que hayan pasado de una cuenca fluvial a otra con suma rapidez sugiere hipótesis alternas de dispersión. En este contexto, la más verosímil sería una dispersión a través de las capas freáticas que conectan las cuencas fluviales entre sí. La presencia temporal de especies epigeas en hábitats hipogeos (caso de Crenobia alpina, Polycelis felina, Phagocatta vitta,...) sugiere esta ruta de dispersión como factible. El uso de esta ruta sería más adecuado para aquellas especies más o menos reófilas, que sean estenotermas de aguas frías, o euritermas, y con tasas de decrecimiento lentas. En este contexto, especies como Polycelis felina, Dugesia $(G)$ tigrina y Dugesia (D) gonocephala parecen las más adecuadas.

\section{COMPETENCIA INTERESPECIFICA Y DESPLAZAMIENTO DE ESPECIES}

Aparte de factores climáticos y tectónicos que han influido en el patrón general de distribución de especies de tríclados, otros factores, como la competencia por el alimento, la adaptación (mayor 0 menor tasa de crecimiento y decrecimiento) a las fluctuaciones de temperatura, la velocidad de la corriente en los cursos de agua y las estrategias reproductivas, han tenido un papel importante para explicar aspectos más circunscritos como es la ausencia o la presencia de una determinada especie o el patrón 
espacial de su distribución (BALL \& REYNOLDSON, 1981).

De estos factores, la competencia interespecifica por el alimento parece determinar la diversidad de especies, la abundancia de cada una de ellas, y la dinámica temporal de su población. El hecho de que varias especies puedan coexistir en determinados lagos de productividad mediana $o$ alta, permite suponer (tal como así sucede) que cada especie de planarias consume con preferencia determinado tipo de presas ("refugio alimenticio" sensu Reynoldson), y que sólo en hábitats poco productivos o en determinadas condiciones ambientales (escasez estacional de alimento, exp̣losión poblacional, polución...). una especie predomina sobre las demás (Ver BALL \& REYNOLDSON, 1981, para referencias bibliográficas)

Los estudios de esta índole en España son inexistentes, y dado que no existen ni lagos ni cursos de agua muy productivos donde puedan coexistir varias especies (en la realidad el número máximo de especies coexistentes ha sido de 2), hemos de convenir que este tipo de estudios es harto problemático. A nuestro juicio, sería necesario en primer lugar, y como tema preliminar, estudiar la dinámica poblacional de cada especie. Más adelante, y como ejemplo de competencia interespecífica sería muy interesante estudiar el impàcto que la introducción y extensión de la especie inmigrante Dugesia (G) tigrina ha"tenido, tiene y tendrá en la distribución de la especie autóctona de hábitat similar: Dugesia (D) gonocephala. Ambas especies son asexuadas, tienen preferencias alimentarias similares, y son ligeramente reófilas. Observaciones en distintas localidades donde coexisten ambas sugieren el lento desplazamiento de Dugesia $(D)$ gonocephala por Dugesia (G) tigrina, especialmente en aquellos tramos más polucionados y donde la temperatura alcanza valores más altos estacionales. Sin embargo, antes de llegar a la conclusión de que Dugesia (G) tigrina está desplazando a la especie autóctona, es necesario realizar un estudio cuantitativo de este fenómeno.

\section{AGRADECIMIENTOS}

En primer lugar a todas aquellas personas que nos han suministrado material de distintas localidades de la península; muy especialmente las aportaciones de diversos miembros del Departamento de Ecología de la Universidad de Barcelona. La información bibliográfica suministrada por los Dres. I. García-Mas y J.M. Jiménez del Departamento de Zoología de la Universidad Complutense de Madrid nos fue muy útil. Finalmente, nuestra más viva gratitud al Profesor Roman Kenk del National Museum of Natural History (Smithsonian Institution, Washington) por la valiosísima información sobre citas antiguas y actuales de planarias en la península y Norte de Africa.

BIBLIOGRAFIA

ALVAREZ, J. \& SELVA, D. 1968. Observaciones sobre invertebrados dulciacuícolas de los altrededores de Madrid. Bol. Real. Soc. española Hist. nat. $65:$ 171-197.

ARDNT, W. 1926. Spongilliden und Turbellarien, aus dem nördlichen und östlichen Spanien, gesammelt von Dr. F. Hass in den Jahren 19141919. Seckenbergiana 8 (1): $22-30$
BÄGUÑ̇̇, J.; SALÓ, E. \& ROMERO, R. 1980. Les planàries d'aigües dolces a Catalunya i les illes Balears. I. Clau sistemàtica i đistribució geogràfica. Bull. Inst. Cat. Hist. Nat. 45: 15-30.

BAGUÑà, J.; SALÓ, E. \& ROMERO, R. 1981. Microdispersió i especiació de planàries d'aigües dolces a la Mediterrània Occidental: el paper de la fragmentació i la migració de microplaques. 
Treb. Ins. Cat. Hist. Nat. 9: 23-38.

BALL, I.R. 1969. An annotated checklist of the freshwater Tricladida of the Neartic and Neotropical regions. Canc. J. Zool. 47: 59-64.

1970. Freshwater Triclads (Turbellaria, Tricladida) from the Oriental region. Zool. J. Limn. Soc., 49: 271-294.

1971. Systematics and biogeographical relationships of some Dugesia species (Tricladida, Paludicola) from Central and South America. Am. Mus. Novit., 2.472: 1-25.

1974. A contribution to the phylogeny and biogeography of the freshwater triclads (Platyhelminthes: Turbellaria). In: Biology of the Turbellaria (N.W. RISER \& M.P. MORSE, eds.). Libbie H. Hyman Memorial Volume. MoGraw-Hill, New York, págs. 339-401.

1974c. A new genus and species of freshwater planarians from Australia (Platyhelminthes: Turbellaria). J. Zool. Lond. 174: 149-158. $1977 \mathrm{~b}$. On the phylogenetic classification of aquatic planarians. Acta. Zool. Fenn. 154: 2135 .

BALL, I.R. \& REYNOLDSON, T.B. 1981. British Planarians. Cambridge Univ. Press. Cambridge.

BEAUCHAMP, P. DE. 1932a. Bioespeológica, LVI (en realidad LXVIII): Turbellariés, Hirudinees, Branchiobdellidés. Deuxième Série. Arch. Zool. exp. et. gen. 73: 113-380.

BENAZZI, M. 1955a. Appunti sulla distribuzione del Tricladi in Italia. Boll. Zool. 22: 149-164.

BENAZZI, M. \& BENAZZI-LENTATI, G. 1976 Animal Cytogenetics. Vol. I. Platyhelminthes págs. 1-182 (B. JOHN, ed.). Gebrüder Borntraeger, Berlín.

BENAZZI, M. \& DERI, P. 1980 . Histo-cy tological study of the ex-fissiparous planarian testicles (Tricladida, Paludicola). Monit. Zool. ital. (N.S.) 14:151-163.

BORDÁS, M. 1921. La profase de reducción en la ovogénesis de Dendrocoelum lacteum Oersted. Trab. Mus. nac. Cienc. nat. (Madrid), Ser. Zool. 44: 101

BORELLI, A. 1905. Sulla presenza della Planaria alpina e della Polycelis comuta nei Pirenei. Boll. Mus. Zool. e Anat. Comp. Univ. Torino. 20:483.

DAHM, A.G. \& GOURBAULT, N. 1978. Tricladida et Temnocephalida (Turbellaria). In: Limnofauna Europea (J. ILLIES, ed.). 2nd ed. Gustav Fischer, Stuttgart.

GAMO, J. \& GOURBAULT, N. 1981. Présence du Turbellarié néarctique Dugesia tigrina (Girard) en Espagne. Bull. Mus. natn. Hist. nat. París. 4e. sér. 3: 147-150.

GAMO, J. \& RODRIGUEZ, F. 1981. Dugesia gonocephala (Turbellaria, Tricladida) nuevo hopedador para Sieboldiellina planarianum (Cilliata, Holotricha, Astomata). Rev. Iber. Parasitol, 41: 313-315.

GOURBAULT, N. 1971a. Turbellariés Triclades des eaux souterraines d'Europe occidentale: nouvelles données géonémiques. Annal. de. Speleol. $26: 181-188$

GOURBAULT, N. 1981. The kary otypes of Dugesia species from Spain (Turbellaria, Triciadida). Hydrobiologia, 84: 45-52.

GOURBAULT, N. \& BENAZZI, M. 1979. Une nouvelle espèce ibérique du groupe "Dugesia gonocephala" (Turbellariés, Triclades). Bull. Mus. natn. Hist. nat. París. 4e sér. 1: 329-337.

HALLEZ, P. 1894b. Catalogue des Rhabdocoelides, Triclades \& Polyclades du Nord de la France. $2 \mathrm{e}$ ed. Lille, 239 págs.

HARTOG, C. DEN 1962. De Nederlandese Platwormen (Tricladida). Wet. Meded. $K$. ned. naturk. Veren. $42: 1-40$.

HOFFMANN, J.A. 1964b. Faune des Triclades Paludicoles du Gran-Duché de Luxembourg. Arch. Inst. G. D. Luxemburg. Sect. Sci. nat. phys. mat., N. Sér. 30: 181-261.

KAWAKATSU, M. 1965. On the ecology and distribution of freshwater planarians in the Japanese islands, with special reference to their vertical distribution. Hydrobiologia 26: 349 408.

KENK, R. 1974. Index of the genera and species of the freshwater Triclads (Turbellaria) of the world. Snith. Contr. Zool. 183: 1-90.

1976. Freshwater planarians (Turbellaria) of North America. U.S. Environmental Protection Agency, Cincinnati, Ohio. 81 págs.

LUTHER, A. 1961. Die Turbellarien Ostfennokandiens. II: Tricladida. Fauna. fenn. 11: 1-42.

MARGALEF, R. 1955. Contribución al estudio de la fauna de las aguas duices del noroeste de España. Publ. Inst. Biol. Aplic. 21 : 137-171.

MEYNELL, P.J, 1973. A hydrobiological survey of a small Spanish river grossly polluted by oil refinery and petrochemical wastes. Freshwater. Biol. 3: 503-520.

OTAMENDI, J.; AGUINAGALDE, A. \& MOYA, J. 1977. Estudio del epitelio de la faringe de los Turbelarios Trícladidos. I. Microscopía electrónica de transmisión. III Bienal. Real. Soc. Españ. Hist. Nat., Granada. Resumen.

PATTEE, E. \& GOURBAULT, N. 1981. Turbelariés Triclades Paludicoles (Planaires d'eau douce). Bull. mens. Soc. Limn. Lyon. 50: 279-304.

REYNOLDSON, T.B. 1967. A key to the British species of freshwater Triclads. Freshwater. biol. Ass., Sci. Publ. 23: 1-38.

STANKOVIC, C. 1960. The Balkan Lake Ohrid and its living world. Den Haag: Dr. W. Junk. 357 págs.

VANDEL, A. 1951. Sur la présence de la planaire Polycelis felina (Dalyell) (= comuta Johnson) dans la Sierra Nevada (Espagne). Bull. Soc. Zool. France. LXXVI, 5-6: 333-335.

VRIES, E.J, DE \& BALL, I.R. 1980. On Dugesia gonocephala from Western Europe. Bijdr. Dierk. 50: $342-350$. 
YOUNG, J.O. 1976. The freshwater Turbellaria of the African Continent. Zool. Anz., Jena 516: $419-432$.

ZABUSOVA, Z.I. 1970. Rasselenie Planaric po so- vetskomu soinzu (Distribution of planarians in the Soviet Union). In: Voprosy evoliutsionnoi morfologii $i$ biotsenologii. Kazanskii Universitet, Kazan, págs. 164-172.

\section{APENDICE}

Lista de localidades en España (hasta 31.12.1982) en las que se han encontrado turbelarios de agua dulce. Para cada especie se indica la localización en tres zonas diferentes de España agrupadas por la intensidad de muestreo, cuando se omite alguna de estas zonas en una especie es que no existen citas por el momento. Además de la localización aproximada se da, para cada cita, la provincia y la fecha de captura o en su defecto la referencia bibliográfica a la que se ha acudido.

\begin{tabular}{|c|c|c|}
\hline \multirow{2}{*}{\multicolumn{3}{|c|}{ Familia DUGESIIDAE }} \\
\hline \multicolumn{2}{|l|}{ Género Dugesia } & \\
\hline \multicolumn{3}{|l|}{$\begin{array}{l}\text { Genero Dugesia } \\
\text { Subgénero Dugesia }\end{array}$} \\
\hline \multicolumn{3}{|l|}{$\begin{array}{l}\text { Dugesia (D) gonocephala } \\
\text { CATALUNYA }\end{array}$} \\
\hline - BAGUNYA et al. 1980 & 9 localidades & \\
\hline - río abajoe, embalse de Sta Fe & Bacelona & $19 / 9 / 80$ \\
\hline - riera de Mura & Barcelona & $15 / 3 / 82$ \\
\hline - rio Negre & Lleida & $23 / 2 / 81$ \\
\hline \multicolumn{3}{|l|}{ BALEARS } \\
\hline - BAGUNYA et al. 1980 & 10 Localidades & \\
\hline - Font de Can Salas, Pollença & Mallorca & GOURBAULT 1981 \\
\hline - Font d'Es Cubelis & Eivissa & $31 / 12 / 82$ \\
\hline - Font de Can Grau & Eivissa & $31 / 12 / 82$ \\
\hline - Pou de Can Plana & Eivissa & $31 / 12 / 82$ \\
\hline $\begin{array}{l}\text { RESTO DE ESPAÑA } \\
\text { - río Guadalete, Algodonales } \\
\text { - fuente de Deifontes }\end{array}$ & $\begin{array}{l}\text { Cádiz } \\
\text { Granada }\end{array}$ & $\begin{array}{r}\text { GOURBAULT } 1981 \\
\text { GOURBAULT \& } \\
\text { BENAZZI } 1979\end{array}$ \\
\hline - río Guadalfeo, Velez de Benandalla & Granada & GOURBAULT 1981 \\
\hline - San Nicolás & Gran Canaria & $\begin{array}{l}\text { BENAZZI \& } \\
\text { DERI } 1980\end{array}$ \\
\hline - río Aliendre & Guadalajara & $\begin{array}{r}\text { GAMO \& } \\
\text { RODRIGUEZ } 1981\end{array}$ \\
\hline - Río Altos de Roldán & Huesca & $15 / 4 / 77$ \\
\hline - río Aceña & Madrid & $21 / 12 / 79$ \\
\hline - río Cofío (cerca de Valdemaqueda) & Madrid & $26 / 1 / 80$ \\
\hline - río Turón (El Burgo, Ronda) & Málaga & GOURBAULT 1981 \\
\hline - río Guadalhorce, Alora & Málaga & GOURBAULT 1981 \\
\hline - río Guadalhorce & Málaga & $\begin{array}{l}\text { BENAZZI \& } \\
\text { deri } 1980\end{array}$ \\
\hline - río Salia, La Viñuela & Málaga & GOURBAULT 1981 \\
\hline - riachuelo cerca de Abanilla & Murcia & $24 / 4 / 76$ \\
\hline - río Pas, cerca de Barcenilla & Santander & $10 / 9 / 82$ \\
\hline - afluente del Eresma, Trescasas & Segovia & $\begin{array}{r}\text { GOURBAULT \& } \\
\text { BENAZZI } 1979\end{array}$ \\
\hline
\end{tabular}


- río Rambla del Mal Burgo, Fortunate Teruel

GOURBAULT \&

BENAZZT 1979

$5 / 10 / 79$

$5 / 10 / 79$

- Fuencaliente, Requena

Valencia

Valencia

Dugesia (D) sicula

BALEARS

- BAGUNYA et al. 1980

2 localidades

Dugesia (D) iberica

BALEARS

- Font de la "coca dels estudiants" Mallorca

GOURBAULT \& BENAZZI 1979

RESTO DE ESPAÑA

- río Júcar, Villalba de la Sierra Cuenca

GOURBAULT \&

BENAZZI 1979

Subgénero Girardia

Dugesia (G) tigrina

CATALUNYA

- BAGUNYA et al. 1980

11 localidades

- Torrent de Santa Fè, Gualba de Baix Barcelona

$6 / 7 / 81$

- Riera de Mura, afl. del Lobregat

Barcelona

$15 / 3 / 82$

- río Ortina, cerca de Vilajuiga

Girona

$18 / 9 / 82$

- río Muga, Castelló d'Empúries

Girona

$18 / 9 / 82$

- embalse de Sta Anna

Lleida

$20 / 6 / 75$

RESTO DE ESPAÑA

- embalse de Amadorio

Alicante

1975

- embalse de Orellana

Badajoz

1975
1975

- embalse de Arrocampo

Cáceres

- embalse de Retortillo

Córdoba

1975

1975

- embalse de Santillana

Madrid

GAMO \&

- río Henares, cerca de Azuqueca de H. Madrid

GOURBAULT 1981

Familia PLANARIIDAE

Género Crenobia

Crenobia alpina

CATALUNYA

- BAGUNYA et al. $1980 \quad 1$ localidad

- Torrent de la Fou de Bor. Lleida

$13 / 8 / 81$

- Torrent de l'Ingla de Coll de Pendís Lleida

$13 / 8 / 81$

RESTO DE ESPAÑA

- río Ara $(2.200 \mathrm{~m})$

- sima Ibón Izagra, Aisa

Huesca

Huesca

Huesca

Huesca

- rincón de Alano, Ansó

Santander

RORELLI 1905

$13 / 7 / 80$

$6 / 8 / 80$

$11 / 8 / 80$

$20 / 5 / 79$

San Pedro de Soba 
Género Polycelis

Polycelis felina

CATALUÑ A

- BAGUNYA et al. 1980

- Torrent de Sta Fè del Montseny

- río Tartres de Guils, Malniu

- río Vadarrós, Vall D'Aran

- río Escrita, cerca de Espot

- río de la Llosa, cerca de Martinet

- río de la Llosa, cerca de Coborriu

19 localidades

$6 / 7 / 81$

Girona

$21 / 8 / 82$

Lleida

$11 / 4 / 82$

Lleida

$10 / 8 / 82$

Lleida

$11 / 8 / 82$

RESTO DE ESPAÑA

- río Trubia, cerca de Bárzana

Lleida

- fuente, San Andrés de Teixido

- fuentes del río Cuervo (afl. del Tajo), cerca de Tragacete

Asturias

$30 / 7 / 81$

La Coruña

$28 / 7 / 81$

- fuente, cara $N$ del Puerto del Lobo (Sierra Nevada)

Cuenca

COURBAULT 1981

- riachuelo, ladera del Pico de S. Juan (Sierra Nevada)

- riachuelo en el collado de Trevelez (Sierra Nevada)

- fuente, loma del Mulhacén

- riachuelo, valle alto del Trevelez

- río Frío

- arroyo de las Truchas (afl. río Cabrillas). cerca de Checa

- Baños de Benasque

- cueva del Veral, Ansó

- río Aragón, $1 \mathrm{~km}$ de Jaca

- río Esera, cerca de Sahún

- río Aguas Limpias, Sallent de Gallego

- lago Batiselles, valle de Astós

- fuentes del río Lobos (afl. del Duero), cerca de Burgo de Osma

- arroyo cerca de la laguna Suelta, Sierra Secundera

Granada

VANDEL 1951

Granada

VANDEL 1951

Granada

Granada

Granada

Granada

Guadalajara

Huesca

Huesca

Huesca

Huesca

Huesca

Huesca

Soria

Zamora

VANDEL 1951

VANDEL 1951

VANDEL 1951 GOURBAULT 1981

$24 / 5 / 80$

ARDNT 1926

$10 / 8 / 71$

$1.4 / 8 / 72$

$22 / 7 / 79$

$8 / 2 / 81$

$11 / 8 / 82$

GOURBAULT 1981

MARGALEF 1955

Polycelis nigra

CATALUNYA

- BAgUNYA et al. 1980

- rio Muga, Perelada - Vilabertran

1 localidad

Girona

$30 / 7 / 80$

- rec Sirvent, cerca de Riumort

Girona

$5 / 9 / 82$

Género Phagocata

Phagocata vitta

CATALUÑA

- BAGUNYA et al. 1980

2 localidades

NOTA DEL EDITOR:

Además de los trabajos que se publican en el presente volumen, se presentaron y fueron leídos en el mismo otros 16 trabajos cuyos resúmenes figuran a continuación. 